\title{
Noninvasive Evaluation of Coronary Perfusion by Transthoracic Doppler Echocardiography in Patients with Anterior Myocardial Infarction before Coronary Intervention
}

\author{
SABINDRA BHUPAL MALLA, MOSTASHIRUL HAQUE, DMMF OSMANY, TANJIMA PARVIN, MOHAMMAD \\ SAFIUDDIN, SAJAL KRISHNA BANERJEE, SYED ALI AHSAN, \\ CHAUDHURY MESHKAT AHMED, KHALED MD IQBAL \\ Department of Cardiology, Bangabandhu Sheikh Mujib Medical University, Dhaka
}

Address of Correspondence: Dr.Sabindra Bhupal Malla, Department of Cardiology, Bangabandhu Sheikh Mujib Medical University, Dhaka, E-mail: sabin_2002@hotmail.com

\begin{abstract}
:
Background: Transthoracic Doppler Echocardiography (TTDE) has made a breakthrough in noninvasive evaluation of coronary artery flow, especially in the distal left anterior descending coronary artery (LAD). The present study was intended to test the hypothesis whether TTDE can differentiate coronary perfusion with Thrombolysis in Myocardial Infarction (TIMI) grade 3 from TIMI grade $\leq 2$ in patients with anterior myocardial infarction (AMI).

Methods: A total of 30 consecutive patients suggestive of anterior MI including those who received thrombolysis with the potential need for Percutaneus Coronary Intervention (PCI) were enrolled in the study. The coronary perfusion was evaluated by antegrade flow visualization in distal LAD by Colour TTDE and antegrade flow velocity measured by pulsed TTDE followed by coronary angiogram for TIMI grade flow in culprit artery. The sensitivity, specificity and diagnostic accuracy were determined by comparing TTDE parameters with angiographic TIMI flow grade.

Results: The antegrade distal LAD flow visualization by Color TTDE enabled differentiation of TIMI 3 from $T I M I \leq 2$ with a sensitivity, specificity, PPV, NPV and diagnostic accuracy of $100 \%, 62.5 \%, 40 \%, 100 \%$ and 70\% respectively. The Receiver Operating Characteristic (ROC) curve constructed to find the cut-off value for antegrade flow velocity in distal LAD in differentiating TIMI 3 from TIMI 0-2 was found to be $30.5 \mathrm{~cm} /$ $s$ with an area under the curve being $0.893, p=0.011$. The antegrade flow velocity in distal LAD $e$ " 30.5 $\mathrm{cm} / \mathrm{s}$ by pulsed TTDE had a fair sensitivity (66.7\%), but had high specificity (100\%) and overall diagnostic accuracy (86.7\%).

Conclusion: The study concluded that antegrade distal LAD flow visualization by Color TTDE can fairly

differentiate TIMI 3 from TIMI $\leq 2$ with high sensitivity, and moderate specificity and diagnostic accuracy. Additionally, the antegrade flow velocity in distal LAD by pulsed TTDE has a moderate sensitivity and high specificity to predict the angiographic TIMI flow grade. Thus, TTDE enables noninvasive differentiation of coronary perfusion in patients with anterior MI before coronary intervention.
\end{abstract}

Key words: \% Transthoracic Doppler Echocardiography \% Myocardial Infarction \% Perfusion.

University Heart Journal 2019; 15(1): 22-27

Introduction

Suboptimal coronary reperfusion with Thrombolysis in Myocardial Infarction (TIMI) grade $\leq 2$ in acute $\mathrm{MI}$ adversely affects patient prognosis and requires additional procedures, such as thrombolysis and/or percutaneous coronary intervention (PCI). ${ }^{1}$ Quick and accurate evaluation of coronary reperfusion in patients with Acute MI is essential to determine whether thrombolysis and/or PCI is required to achieve reperfusion. ${ }^{2}$ Although currently available noninvasive tools that evaluate coronary reperfusion, including the resolution of ST-segment elevation and the rapid washout of serum cardiac biomarkers, are accurate, they are indirect and require serial evaluation requiring 90 minutes to several hours. ${ }^{1}$

Although Coronary angiography as a standard tool is available, they are not feasible for widespread use in developing countries like Bangladesh due to their high cost and availability limited in advanced urban 
medical centers. Several tools including coronary sinus thermodilution, cardiac nuclear imaging, inert gas wash-out method, cardiac magnetic resonance, and intracoronary doppler flow wire, have already measured coronary flow and coronary flow reserve (CFR) for decades. However, these methods, although helpful for research, have limited clinical implications, for they are complex, time-consuming, expensive, not easily available, or invasive. Thus, a rapid noninvasive diagnosis of coronary perfusion is important for decision making. ${ }^{3}$

Transthoracic Doppler echocardiography (TTDE), to measure coronary flow and coronary flow velocity (CFV), is a non-invasive procedure. It is easily available at bedside, inexpensive, and has multiple potential clinical applications without radiation exposure. As the feasibility of TTDE to evaluate coronary perfusion has not been widely investigated, the purpose of the present study was to establish this by comparing the TTDE findings of distal LAD flow with

TIMI grades by angiography in patients with anterior myocardial infarction.

The clinical implication of the study will be, if the patient has poor coronary perfusion as evidenced by absent antegrade flow in colour TTDE and low flow velocity in Pulse TTDE, then he/she is the candidate for revascularization. Thus the study will help us in guiding further treatment strategy. It also helps in determining the prognosis of the patient with anterior MI by differentiating coronary perfusion with TIMI 3 from TIMId" 2. It also has additional benefit by deferring the significant number of patients with good perfusion from undergoing invasive procedure. It will also prevent the hazards of invasive procedure in significant number of MI patients, as well as be easily available and affordable for the general public of developing countries like Bangladesh.

\section{Patients and methods}

\section{Study design}

The present study was analytical, a cross-sectional study. The study was carried out in the Department of Cardiology, University Cardiac Centre, Bangabandhu Sheikh Mujib Medical University (BSMMU), Dhaka over a period of 13 months between August 2017 to September 2018. Patients presenting with signs and symptoms of MI for evaluation were the study population.A total of 30 patients with the following inclusion and exclusion criteria were enrolled: Patients with acute anterior MI presenting with-
- Typical chest pain lasting $>30$ minutes and $\leq 12$ hours from onset, not relieved by standard antianginal therapy.

- $\quad$ ST segment elevation e $\geq 0.2 \mathrm{mV}$ in male and $\geq 0.15 \mathrm{mV}$ in female in precordial leads.

- Subsequent increase in Troponin I and creatinine kinase $\geq 2$-times the upper limit of normal.

- Patients with acute anterior MI who received thrombolysis with the potential need for Percutaneus Coronary Intervention (PCI).

- Patient with recent and old anterior MI with the potential need for Percutaneous Coronary Intervention (PCI)

- Patients with low systolic blood pressure (dd $80 \mathrm{~mm}$ $\mathrm{Hg}$ ) were excluded from the study.

\section{Detailed procedure:}

All patients with cardiac chest pain attending at UCC, BSMMU were selected as case on the

basis of eligibility criteria.On the basis of history, examination and investigations (ECG and Cadiac biomarkers) all patients with anterior MI were selected including those who received thrombolysis. Among these patients, who were under going PCI under guideline-based indications were eligible for the study. The eligible patients were explained about the nature and purpose of the study and those who provided informed written consent participated in the study. All participants underwent emergent TTDE and subsequent coronary angiography.

\section{Transthoracic Doppler Echocardiography:}

Standard 2-dimensional and Doppler echocardiography was performed in the supine or left lateral decubitus position with a digital ultrasound system. After the standard examination, distal LAD flow was evaluated with a 4- to 7-MHz transducer. The transducer was placed at the fourth or fifth intercostal space between the cardiac apex and the parasternal area, such that the anterior interventricular groove was visualized in the short-axis view. Then the transducer was rotated to visualize the long axis of the groove, and color TTDE with a Nyquist limit of \pm 19 to $\pm 24 \mathrm{~cm} / \mathrm{s}$ was applied to visualize the LAD flow with relatively low velocity; its velocity was measured by pulsed TTDE. When the distal LAD flow was retrograde by color doppler or was not visualized within 5 minutes, antegrade LAD flow was considered to be absent, and the TTDE coronary study was discontinued. 

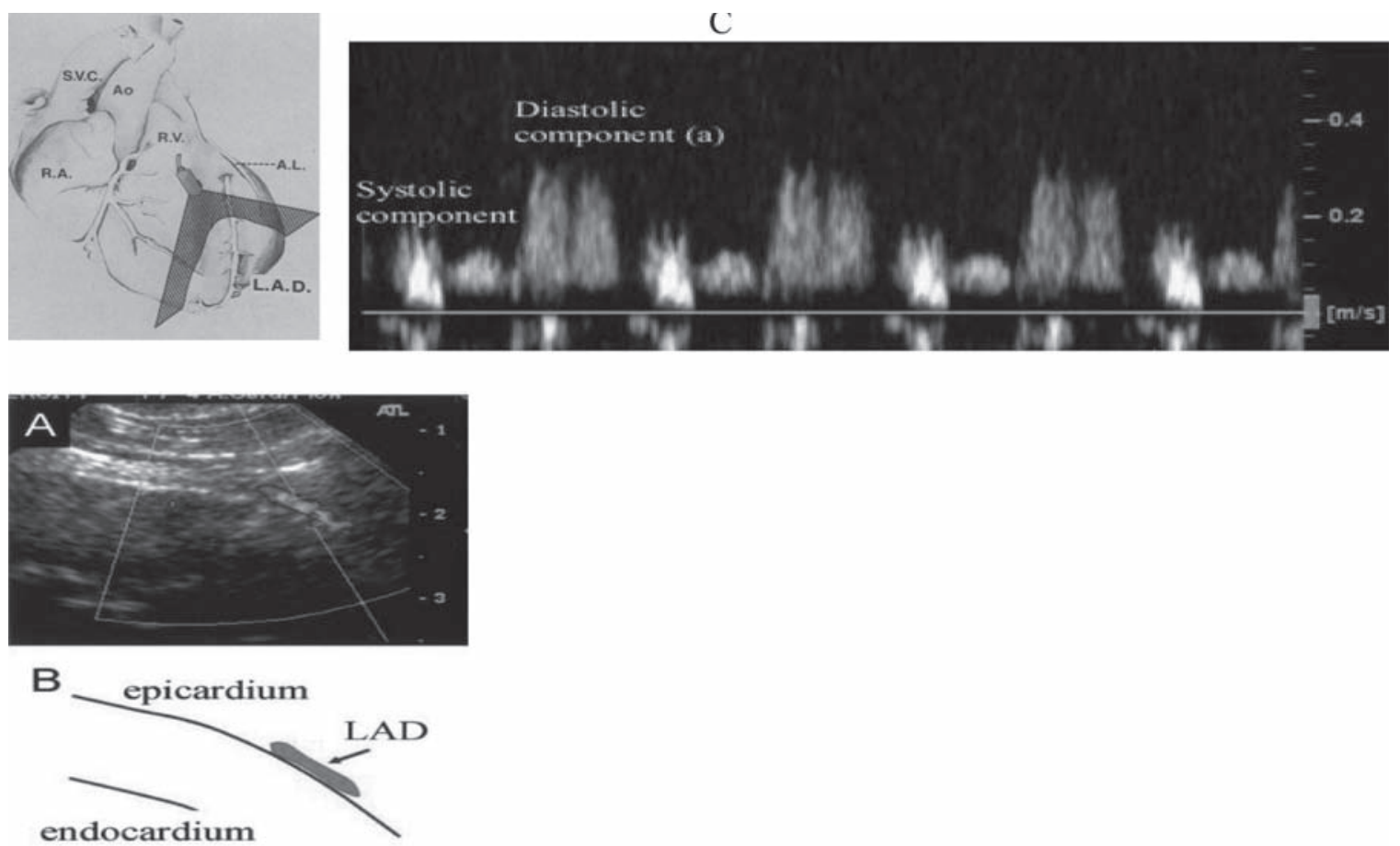

Fig.-1: A and B, Distal LAD flow visualized by Colour TTDE. C, Phasic distal LAD flow velocity by pulsed TTDE

Observers blinded to the patients' data reviewed the TTDE recordings. As pulsed Doppler parameters of LAD flow, the peak velocity was measured with an analyzer incorporated into the ultrasound system. These parameters were calculated for 3 consecutive beats and averaged.

So, by Colour TTDE, patients with visual antegrade distal LAD flow and without distal LAD flow were determined.By Pulsed TTDE, LAD flow velocity was measured in all patients who had visual flow by Colour TTDE.

\section{Coronary Angiography}

Coronary angiography was performed immediately after the TTDE study. Several views of the

LAD were digitally acquired, and the percent diameter stenosis were quantified with a commercially available system with a $6 \mathrm{~F}$ catheter as the reference. The angiographic TIMI grade (Grade 0,1,2 or 3) were evaluated with coronary angiography. The angiographic TIMI grade of the LAD was evaluated using a cardiac review station equipped with a frame counter. The frame rate was 30 frames/s. After completion of the data collection, the angiographic TIMI findings were compared with the TTDE findings (distal LAD flow and velocity) and evaluated for the presence of statistical significance. The sensitivity, specificity, positive and negative predictive values and the accuracy of TTDE by
Colour Doppler and pulse wave doppler as diagnostic tool to detect the coronary perfusion, i.e, whether TIMI 3 or TIMI $£ 2$ in anterior myocardial infarction were evaluated.

\section{Data analysis}

Data were processed and analyzed using computer software SPSS (Statistical Package for Social

Sciences) version 16 (SPSS Inc., Chicago, IL, USA). The results were expressed with corresponding percentage, mean/median and standard deviation from the mean/ median. The ability of peak LAD flow velocity to indicate TIMI 3 was analyzed by receiver operating characteristic curves. The diagnostic accuracy, sensitivity and specificity of antegrade flow visualization and flow velocity by TTDE in predicting coronary perfusion were determined by comparing with angiographic TIMI flow grade.

\section{Results}

The baseline characteristics of the patient is shown in Table I. In the present study Colour TTDE visualized antegrade flow in distal LAD in half $(50 \%)$ of the cases and the mean flow velocity was 26 (range: $13-49$ ) cm/ s. Coronary angiographic findings revelaed that $22(73.3 \%)$ of the 30 patients had significant LAD stenosis. The distribution of TIMI flow grade was classified as TIMI-0 (36.6\%), TIMI-1 (26.7\%), TIMI-2 (16.7\%) and TIMI-3 (20\%). 
Table-I

Patient Characteristics

\begin{tabular}{|c|c|c|c|}
\hline & Frerquency & Percentage & Mean \pm SD (range) \\
\hline Age (years)d" 40 & 2 & 6.7 & \\
\hline $41-50$ & 10 & 33.3 & \\
\hline$>50$ & 18 & 60.0 & $53.3 \pm 9.2$ years \\
\hline Male & 22 & 73.3 & $(33-70)$ \\
\hline FemaleAnginaTypical & 826 & 26.786 .7 & \\
\hline Atypical & 4 & 13.3 & \\
\hline Family history of CAD & 19 & 63.3 & \\
\hline Overweight or Obese & 11 & 36.7 & \\
\hline Hypertension & 27 & 90.0 & \\
\hline Diabetes & 26 & 86.7 & \\
\hline DyslipidemiaSmokerNon-smoker & 248 & 80.026 .7 & \\
\hline Current & 12 & 40.0 & \\
\hline Previous & 10 & 33.3 & \\
\hline Chronic Kidney DiseaseCPK MB (IU/ml) & 3 & 10.0 & $108.4 \pm 54.9(33-323)$ \\
\hline $\begin{array}{l}\text { Troponin I (ng/ml)Intravenous Thrombolysis } \\
\text { (Streptokinase) }\end{array}$ & 4 & 13.3 & $4.6 \pm 2.5(0.02-10)$ \\
\hline Pulse (bpm) & & & $83 \pm 10(64-102)$ \\
\hline Systolic BP(mm Hg) & & & $125.8 \pm 15.8(90-146)$ \\
\hline Diastolic BP (mm Hg) & & & $76.9 \pm 10.6(60-96)$ \\
\hline ST elevation & 26 & 86.7 & \\
\hline Presence of Q wave & 13 & 43.3 & \\
\hline \multicolumn{4}{|l|}{ Colour TTDE } \\
\hline \multicolumn{4}{|l|}{ (Antegrade flow in distal LAD) } \\
\hline Visualised & 15 & 50 & \\
\hline Not visualized & 15 & 50 & \\
\hline \multicolumn{4}{|l|}{ Pulse TTDE } \\
\hline Velocity in distal LAD (cm/s) & & & $6.0 \pm 9.6(13-49)$ \\
\hline \multicolumn{4}{|l|}{ CAG } \\
\hline Significant stenosis in LAD & 22 & 73.3 & \\
\hline \multicolumn{4}{|l|}{ TIMI grade } \\
\hline 0 & 11 & 36.6 & \\
\hline 1 & 8 & 26.7 & \\
\hline 2 & 5 & 16.7 & \\
\hline 3 & 6 & 20.0 & \\
\hline
\end{tabular}

CAD, coronary artery disease; CPK MB, creatinine phosphokinase myocardial band; BP, blood pressure; TTDE, transthoracic doppler echocardiography; LAD, left anterior descending artery; TIMI, thrombolysis in myocardial infarction.

\section{Accuracy of Colour TTDE in predicting coronary perfusion (TIMI flow)}

The accuracy of antegrade flow visualization in distal LAD by Colour TTDE in predicting coronary perfusion is shown in Table 2. The sensitivity of the test in diagnosing TIMI-3 was $6 / 6$ x $100=100 \%$ and specificity of the test in correctly differentiating TIMI $\leq 2$ from those who had TIMI 3 was $15 / 24$ x $100=62.5 \%$. The positive and negative predictive values of the test were

$6 / 15 ? 100=40 \%$ and $15 / 15 ? 100=100 \%$ respectively. The overall diagnostic accuracy of the test is $(6+15) /(6$ $+9+0+15) \times 100=70.0 \%$ (Table 2). 
Table-II

Accuracy of Colour TTDE in predicting coronary perfusion

\begin{tabular}{lccc}
\hline $\begin{array}{l}\text { Antegrade flow } \\
\text { in distal } \\
\text { LAD }\end{array}$ & $\begin{array}{l}\text { CAG } \\
\text { TIMI 3 } \\
(\mathrm{n}=6)\end{array}$ & $\begin{array}{l}\text { TIMI } \leq 2 \\
(\mathrm{n}=24)\end{array}$ \\
\hline Visualised & 6 & 9 & 15 \\
Not visualized & 0 & 15 & 15 \\
\hline Total & 6 & 24 & 30 \\
\hline
\end{tabular}

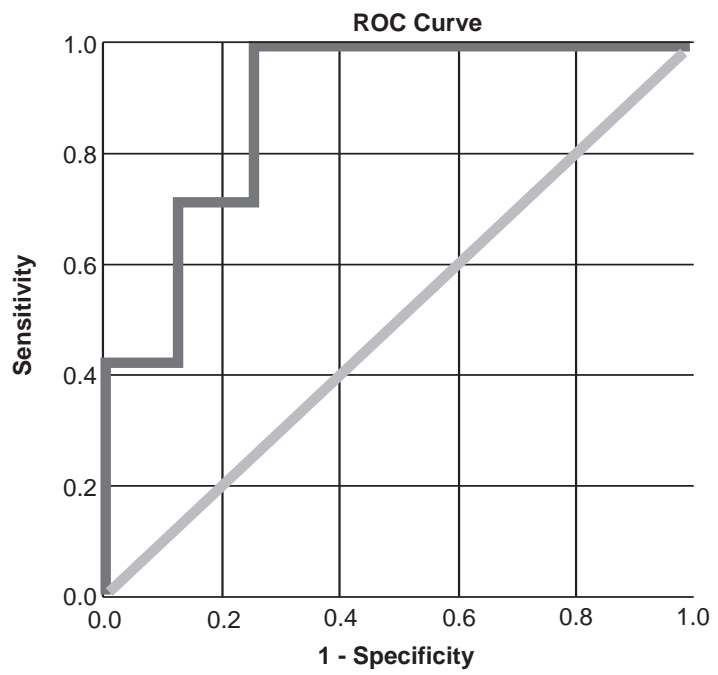

Fig.-2: Area under the ROC curveAccuracy of Pulsed TTDE in predicting TIMI flow.

Before determining the diagnostic accuracy of antegrade flow velocity in differentiating angiographic TIMI 3 flow from TIMI $\leq 2$, an optimum cut-off value for antegrade flow velocity was to be found out using Receiver Operating Characteristic (ROC) curve (Fig 1). The best cut- off value for optimum sensitivity (42.9\%) and high specificity $(100 \%)$ was 30.5 with an area under the curve being $0.893(95 \% \mathrm{CI}=0.725-1.000), \mathrm{p}=0.011$ meaning $89.3 \%$ of the TIMI 3 flow will be correctly classified with antegrade flow velocity $\geq 30.5 \mathrm{~cm} / \mathrm{s}$.

The accuracy of antegrade flow velocity in distal LAD (at cut-off value of $30.5 \mathrm{~cm} / \mathrm{s}$ ) by Pulsed TTDE in diagnosing coronary perfusion in culprit artery is shown in table 3 . The sensitivity of the test in detecting TIMI-3 was $4 / 6 \times 100=66.7 \%$ and specificity of the test in correctly differentiating TIMI $\leq 2$ from those who had good reperfusion (TIMI 3) was 9/9x100 $=100 \%$. The positive and negative predictive values of the test were $4 / 4 \times 100=100 \%$ and $9 / 11 \times 100=81.8 \%$ respectively.
The overall diagnostic accuracy of the test was $(4+9) /$ $(4+0+2+9) \times 100=86.7 \%$ (Table 3$)$.

Table-III

Accuracy of antegrade flow velocity in distal LAD by pulsed TTDE in predicting coronary perfusion

\begin{tabular}{|c|c|c|c|}
\hline \multirow{2}{*}{$\begin{array}{l}\text { Antegrade flow velocity } \\
\text { in distal LAD }\end{array}$} & \multicolumn{2}{|c|}{ CAG } & \multirow[b]{2}{*}{ Total } \\
\hline & TIMI 3 & $\mathrm{TIMI} \leq 2$ & \\
\hline$\geq 30.5 \mathrm{~cm} / \mathrm{s}$ & 4 & 0 & 4 \\
\hline$<30.5 \mathrm{~cm} / \mathrm{s}$ & 2 & 9 & 11 \\
\hline Total & 6 & 9 & 15 \\
\hline
\end{tabular}

\section{Discussion}

In the present study antegrade distal LAD flow visualization by Color TTDE could fairly differentiated TIMI 3 from TIMI $\leq 2$ with a sensitivity, specificity, PPV, NPV and diagnostic accuracy of $100 \%, 62.5 \%, 40 \%$, $100 \%$ and $70 \%$ respectively. These findings compare well with those of a similar study by Lee et al (2003) who found a sensitivity, specificity, and accuracy of $85 \%$, $67 \%$, and $72 \%$, respectively. Furthermore, the antegrade flow velocity in distal LAD $\geq 30.5 \mathrm{~cm} / \mathrm{s}$ by pulsed TTDE indicates TIMI 3, whereas flow velocity $<30.5 \mathrm{~cm} / \mathrm{s}$ indicates TIMI $\leq 2$ flow. The sensitivity, specificity, PPV, NPV and diagnostic accuracy of the test are $66.7 \%$, $100 \%, 100 \%, 81.8 \%, 86.7 \%$ respectively. Therefore, the findings of the present study seems to be a novel, noninvasive, tool that differentiates anterior MI patients with and without preserved coronary perfusion and thus helps in assessing which patients need additional acutephase procedures before emergent PCI. The ROC curve constructed to find the cut-off value for antegrade flow velocity in distal LAD in differentiating TIMI 3 from TIMI $0-2$ was taken as $30.5 \mathrm{~cm} / \mathrm{s}$ with $100 \%$ specificity, although with low sensitivity $(42.9 \%)$. The rationale behind it is, due to high specificity no cases with low coronary perfusion will be detected as good perfusion which may forbid the patient from receiving appropriate treatment. Furthermore, due to fair sensitivity, patients with good coronary perfusion may be detected as low perfusion in which case further invasive approach (CAG) can detect coronary perfusion and guide appropriate treatment

However, the present study confirmed the feasibility of TTDE to evaluate coronary pathophysiology and further demonstrated its utility to differentiate TIMI 0 to 2 and TIMI 3 perfusion in patients with anterior MI. Therefore, for most patients before PCI, TIMI 3 can be diagnosed 
on the basis of antegrade LAD flow visualization and LAD velocity. However, no- reflow can develop in a small number of patients before PCI,4 with a potential for finding TIMI 2 flow with a preserved diastolic peak velocity even before the PCI. In such patients, the current criteria could present a problem, and in such cases, the diastolic deceleration time or the velocity time integral will likely be useful in differentiating TIMI 3 from TIMI $2.4,5$.

\section{Limitations}

There are several limitations in our study which deserve mention. In the emergent situation, the LAD flow signals by TTDE were occasionally suboptimal. Besides, we did not use any contrast agents to enhance Doppler signals, 6 for the use of contrast agents may be associated with harmful effects. Thus, further technical innovations of TTDE with contrast agents are expected to expand the feasibility of evaluating the LAD and other vessels.6,7

Although the present study demonstrated the feasibility of Pulsed TTDE to differentiate TIMI 0 to 2 from TIMI 3 perfusion in patients with anterior MI before PCI, the criterion of antegrade flow velocity e" $30.5 \mathrm{~cm} / \mathrm{s}$ needs further evaluation in a larger number of patients for the present study was conducted only on 30 patients. In addition, there were some discrepancies between the perfusion evaluated by TTDE and that by angiography. One explanation might be the dynamic nature of coronary reperfusion.8 Recanalization or re-occlusion in the culprit lesion might have developed between TTDE and angiography. In spite of that, the purpose of the present study was achieved by evaluating feasibility of TTDE to differentiate TIMI 3 from TIMI 0 to 2 flow in patients with anterior MI before emergent PCI

\section{References:}

1. Lee S, Otsuji Y, Minagoe S, Hamasaki S, Toyonaga K, Negishi M, Tsurugida M, Toda H, ChuwaTei 2003, 'Noninvasive Evaluation of Coronary Reperfusion by Transthoracic Doppler Echocardiography in Patients With Anterior Acute Myocardial Infarction Before Coronary Intervention,' Circulation, vol. 108, pp. 2763-68.

2. Lee S, Otsuji Y, Minagoe S, Hamasaki S, Toyonaga K, Obata H, Takumi T, Arimura H, Miyata M, Biro S, Toda H. Correlation between distal left anterior descending artery flow velocity by transthoracic Doppler echocardiography and corrected TIMI frame countbefore mechanical reperfusion in patients with anterior acute myocardial infarction. Circulation Journal. 2005;69(9):1022-8.

3. Meimoun P, Tribouilloy C. Non-invasive assessment of coronary flow and coronary flow reserve by transthoracic Doppler echocardiography: a magic tool for the real world. European Journal of Echocardiography. 2008 Feb 19;9(4):449-57.

4. Akasaka T, Yoshida K, Kawamoto T, Kaji S, Ueda Y, Yamamuro A, Takagi T, Hozumi T. Relation of phasic coronary flow velocity characteristics with TIMI perfusion grade and myocardial recovery after primary percutaneous transluminal coronary angioplasty and rescue stenting. Circulation. 2000 May 23;101(20):2361-7.

5. Iwakura K, Ito H, Takiuchi S, Taniyama Y, Nakatsuchi Y, Negoro S, Higashino Y, Okamura A, Masuyama T, Hori M, Fujii K. Alternation in the coronary blood flow velocity pattern in patients with no reflow and reperfused acute myocardial infarction. Circulation. 1996 Sep 15;94(6):1269-75.

6. Voci P, Pizzuto F, Mariano E, Puddu PE, Chiavari PA, Romeo F. Measurement of coronary flow reserve in the anterior and posterior descending coronary arteries by transthoracic Doppler ultrasound. The American journal of cardiology. 2002 Nov 1;90(9):988-91.

7. Caiati C, Zedda N, Montaldo C, Montisci R, Iliceto S. Contrastenhanced transthoracic second harmonic echo Doppler with adenosine: a noninvasive, rapid and effective method for coronary flow reserve assessment. Journal of the American College of Cardiology. 1999 Jul 1;34(1):122-30.

8. Hackett D, Davies G, Chierchia S, Maseri A. Intermittent coronary occlusion in acute myocardial infarction. New England Journal of Medicine. 1987 Oct 22;317(17):1055-9. 